\title{
Simultaneous measurement of cholinergic tone and neuronal network dynamics in vivo in the rat brain using a novel choline oxidase based electrochemical biosensor
}

Ricardo M. Santos ${ }^{\mathrm{a}, \mathrm{c}}$, João Laranjinha ${ }^{\mathrm{a}, \mathrm{b}}$, Rui M. Barbosa ${ }^{\mathrm{a}, \mathrm{b}^{*}}$ and Anton Sirota ${ }^{\mathrm{c}^{*}}$

${ }^{a}$ Center for Neuroscience and Cell Biology, University of Coimbra, 3004-517 Coimbra, Portugal

${ }^{b}$ Faculty of Pharmacy, University of Coimbra, 3000-548 Coimbra, Portugal

${ }^{c}$ Bernstein Center for Computational Neuroscience and Munich Cluster for Systems Neurology (SyNergy), Ludwig-Maximilians Universität München, 82152 Planegg-Martinsried, Germany

*Corresponding authors:

Rui M. Barbosa

Faculty of Pharmacy, University of Coimbra, Health Campus, Azinhaga de Santa Comba, 3000-548

Coimbra, Portugal

Tel. +351239488463

Email address: rbarbosa@ff.uc.pt

Anton Sirota

Ludwig-Maximilians Universität München, Grosshaderner Strasse 2, 82152 Planegg-Martinsried, Germany

Tel. +4989218074805

Fax: +4989218074803

Email address: sirota@biologie.uni-muenchen.de 


\begin{abstract}
Acetylcholine ( $\mathrm{ACh}$ ) modulates neuronal network activities implicated in cognition, including theta and gamma oscillations but the mechanisms remain poorly understood. Joint measurements of cholinergic activity and neuronal network dynamics with high spatio-temporal resolution are critical to understand ACh neuromodulation. However, current electrochemical biosensors are not optimized to measure nanomolar cholinergic signals across small regions like hippocampal sub-layers.

Here, we report a novel oxidase-based electrochemical biosensor that matches these constraints. The approach is based on measurement of $\mathrm{H}_{2} \mathrm{O}_{2}$ generated by choline oxidase (ChOx) in the presence of choline (Ch). The microelectrode design consists of a twisted pair of $50 \mu \mathrm{m}$ diameter Pt/Ir wires (sensor and sentinel), which is scalable, provides high spatial resolution and optimizes common mode rejection. Microelectrode coating with $\mathrm{ChOx}$ in chitosan cross-linked with benzoquinone is simple, mechanically

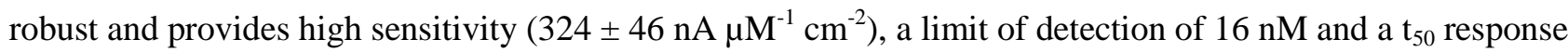
time of $1.4 \mathrm{~s}$.

Local field potential (LFP)-related currents dominate high-frequency component of electrochemical recordings in vivo. We significantly improved signal-to-noise-ratio compared to traditional sentinel subtraction by a novel frequency domain common mode rejection procedure that accounts for differential phase and amplitude of LFP-related currents on the two channels.

We demonstrate measurements of spontaneous nanomolar $\mathrm{Ch}$ fluctuations, on top of which micromolar $\mathrm{Ch}$ increases occurred during periods of theta activity in anesthetized rats. Measurements were not affected by physiological $\mathrm{O}_{2}$ changes, in agreement with the low biosensor $\mathrm{Km}$ for $\mathrm{O}_{2}(2.6 \mu \mathrm{M})$. Design and performance of the novel biosensor opens the way for multisite recordings of spontaneous cholinergic dynamics in behaving animals.
\end{abstract}

Keywords:

Microbiosensor; Acetylcholine; Neuromodulation; Hippocampus; LFP oscillations; I $n$ vivo electrochemistry. 


\section{Introduction}

Acetylcholine (ACh) is a neuromodulator strongly implicated in learning and cognition (Deiana et al., 2011; Hasselmo and Sarter, 2011; Micheau and Marighetto, 2011; Picciotto et al., 2012). In the hippocampus, cholinergic afferent activity modulates multiple aspects of neuronal network function implicated in cognition via modulation of internal dynamics, including theta, gamma oscillations and sharp-waves (Buzsáki, 2002; Pignatelli et al., 2011; Vandecasteele et al., 2014). However, the complexity of cholinergic neuromodulation, partly owing to the ubiquitous expression of ACh receptors in multiple interacting neuron types and to the apparently diffuse patterns of cholinergic innervation in hippocampal and cortical regions has rendered the precise mechanisms of ACh action poorly understood (Buzsaki, 2002; Pignatelli et al., 2012; Teles-Grilo Ruivo and Mellor, 2013). Nevertheless, it has become increasingly recognized that the interaction between the spatio-temporal profile of ACh signals and the neurophysiological and behavioural context in which they occur is critical to shape ACh effects (Leão et al., 2012; Lovett-Barron et al., 2014; Muñoz and Rudy, 2014; Parikh et al., 2007). Therefore, sensitive measurements of cholinergic activity with high spatio-temporal resolution coupled to simultaneous assessment of neuronal network dynamics and behavior are critical to understand ACh neuromodulation in vivo.

Fast electrochemical techniques, when associated with microelectrodes are particularly suitable for monitoring neuromodulators in the brain extracellular space owing to the high sensitivity, selectivity and spatio-temporal resolution that can be achieved with minimal tissue damage (Lama et al., 2012). Additionally, electrochemical recordings carry local field potential (LFP)-related currents (Zhang et al., 2009), thus offering the unique possibility to measure changes in neuromodulator tone together with ongoing neuronal network activity (inferred from LFP features) using a single sensor.

Different types of enzyme-based electrochemical microbiosensors have been used to study cholinergic transmission by measuring extracellular changes in choline (Ch), a product of ACh hydrolysis by acetylcholinesterase (Burmeister et al., 2003; Garguilo and Michael, 1996, 1993; Xin and Wightman, 1997). However, most of them were constrained by high limits of detection (LODs in the micromolar range) and slow responses (decades of seconds). A successful approach relied on immobilization of choline oxidase (ChOx) on the surface of ceramic-based platinum microelectrode arrays (MEAs). The enzyme catalyzes Ch oxidation in the presence of $\mathrm{O}_{2}$, generating $\mathrm{H}_{2} \mathrm{O}_{2}$, which can be detected by electrochemical oxidation on the electrode surface. These sensors exhibited sub-micromolar LODs and response times of few seconds (Burmeister et al., 2003; Parikh et al., 2007, 2004; Zhang et al., 2010). Each Pt site is individually addressable allowing the use of specific sensing chemistry, which is a major advantage of MEAs. Comparison between recordings from sentinel sites (coated with a matrix lacking ChOx) and Cholinemeasuring $(\mathrm{ChOx})$ sites allows identification of current profiles not related with $\mathrm{Ch}$ dynamics (e.g. fluctuations of electroactive interferents like ascorbate or LFP-related currents). Thus, common mode rejection using a sentinel channel greatly improves selectivity and signal-to-noise of biosensor recordings.

The use of MEA-based Ch biosensors in vivo supported the reliability of measuring extracellular $\mathrm{Ch}$ dynamics as an index of local ACh release, provided that acetylcholinesterase activity is stable (Parikh and Sarter, 2013; Parikh et al., 2004). Insights into the link between ACh, neuronal network activity and behavior 
have been provided with much better spatio-temporal resolution than in previous microdyalisis studies. For instance, choline increases in the nanomolar range were measured during theta periods in the CA1 subregion of the hippocampus which lagged theta onset by several seconds (Zhang et al., 2010). In freely behaving rats, micromolar $\mathrm{Ch}$ transients lasting few seconds were recorded in the pre-frontal cortex when animals attended to a cue. These signals occurred on top of slower Ch fluctuations whose slope predicted animal's success in cue detection (Parikh et al., 2007). Thus, choline signals can apparently span multiple time scales and amplitudes ranging from seconds to minutes and from low nanomolar to micromolar, respectively. Characterizing the different types of $\mathrm{Ch}$ dynamics and their associated neurophysiological and behavioral context is therefore of great relevance to understand ACh neuromodulation.

However, the measurement of spontaneous nanomolar Ch fluctuations across small heterogeneous regions like hippocampal sub-layers is technically challenging and requires further optimization of the biosensor design. In particular: a) the size of the recording sites should be small enough to allow resolution of small brain regions like hippocampal sub-layers; b) Enzyme immobilization should be efficient in order to keep good sensitivity at small electrode surfaces; c) Given that in most cortical and dorsal-hippocampal regions the gradient of LFP profiles is much steeper across the dorso-ventral axis, the ChOx and sentinel sites should be located at the same depth in order to optimize common LFP mode rejection using sentinel channel; d) Since complex impedance spectra of $\mathrm{ChOx}$ and sentinel sites are, in general, expected to be different across wide range of frequencies, common mode rejection procedure has to be performed in the complex form in the frequency domain, which should improve signal-to-noise ratio compared to conventional sentinel subtraction; e) Chronic measurement of $\mathrm{Ch}$ dynamics across multiple regions and layers in freely moving animals requires flexible and minimally invasive design of the electrode that can be scaled and individually driven to the target location, similar to conventional stereotrode electrodes for extracellular recordings (McNaughton et al., 1983).

An additional concern, related to the use of oxidase-based biosensors in vivo is their $\mathrm{O}_{2}$ dependence. Measurements of extracellular $\mathrm{O}_{2}$ concentration in the neocortex and hippocampus have shown a broad distribution of $\mathrm{O}_{2}$ basal levels around a mean of 35-50 $\mu \mathrm{M}$ but with a considerable number of observations at lower levels, down to $10 \mu \mathrm{M}$ (Murr et al., 1994; Nair et al., 1987). The extracellular $\mathrm{O}_{2}$ concentration is also dynamically regulated according to changes in metabolic demand imposed by neuronal activity (Lourenço et al., 2014; Masamoto and Tanishita, 2009). Thus, negligible $\mathrm{O}_{2}$ dependence over the range of concentrations described above is a critical requirement for a biosensing application in the brain, particularly when fluctuations in analyte concentration are very small (nanomolar range). Data on $\mathrm{Ch}$ biosensors $\mathrm{O}_{2}$ dependence is scarce, but some results obtained with MEA-based biosensors do not exclude a potential interference when $\mathrm{O}_{2}$ levels are low (Burmeister et al., 2003).

In this work we developed a new choline microbiosensor design for the measurement of spontaneous fluctuations of cholinergic tone, taking into account the limitations described above. The microelectrodes consisted of two side-by-side disk shaped $50 \mu \mathrm{m}$ diameter Pt/Ir wires. The microdisks provide high spatial resolution for measurements within the hippocampus and the orientation of the wires, positioned at the same depth, optimizes sentinel channel subtraction in vivo. 
Choline oxidase was immobilized in a chitosan matrix using $p$-benzoquinone as crosslinker. This novel coating procedure is robust and fast (biosensors can be used one hour after preparation) and provides very good sensitivity considering the small size of the recording sites.

In anesthetized rats, microbiosensor measurements of Ch and LFP-related currents were carried out in the stratum oriens of the CA1 field of hippocampus. Large Ch increases were observed during periods of theta oscillations, which superimposed on the smaller spontaneous nanomolar Ch fluctuations. In vitro and in vivo results indicate that the biosensor response is not affected by physiological $\mathrm{O}_{2}$ changes. The data supports the reliability of the microbiosensors to jointly measure spontaneous cholinergic activity and neuronal network dynamics in the brain. 


\section{Methods}

\subsection{Chemicals and solutions}

All chemicals were of analytical grade and were used as received. Dibasic sodium phosphate hepta-hydrate, monobasic potassium phosphate, sodium chloride and potassium chloride were purchased from Carl Roth. Hydrogen peroxide (30\%), choline chloride, sodium L-ascorbate (AA), uric acid (UA), dopamine (DA), chitosan (low molecular weight), choline oxidase (EC 1.1.3.17) from Alcaligenes sp., bovine serum albumin (BSA), $p$-benzoquinone, Nafion ${ }^{\circledR}$ perfluorinated resin $5 \%$ in lower aliphatic alcohols and water (15-20\%) and urethane were obtained from Sigma-Aldrich. The solutions were prepared in ultra-pure deionized water $(\geq 18 \mathrm{M} \Omega . \mathrm{cm}$ ) from a Milli-Q water purification system. Phosphate-buffered saline (PBS) $0.05 \mathrm{M}, \mathrm{pH} 7.4$ contained: $0.041 \mathrm{M} \mathrm{Na}_{2} \mathrm{HPO}_{4}, 0.009 \mathrm{M} \mathrm{KH}_{2} \mathrm{PO}_{4}, 0.098 \mathrm{M} \mathrm{NaCl}$ and $0.002 \mathrm{M} \mathrm{KCl}$.

\subsection{Microelectrode fabrication}

The microelectrode support material was a $50 \mu \mathrm{m}$ diameter Platinum/Iridium (90/10) wire insulated by a polyimide coating (California Fine Wire Company). To make dual-channel stereotrodes, a single wire loop was hung on a horizontal bar and the bottom of the loop was pulled down by hooking it into a bent needle attached to a metallic bar. The loop was then twisted by rotation of the needle/metallic bar, induced by a magnetic stirrer placed underneath. Next, the twisted wire was moderately heated with a heat gun, which slightly melted the wire insulation, strengthening the connection between twisted wire segments. Finally, the bottom (twisted) and upper (untwisted) endings of the wire were cut resulting in two independent wires in a twisted configuration.

Finally, the electrode wire was inserted in a metal tube leaving a few centimeters of exposed twisted wire on one side and soldering the untwisted endings to gold pins on the other side, for connection to the head-stage. The untwisted parts of the wires were protected by using shrinking tubes for easier manipulation of the microelectrodes. To allow independent modification of the wires, their tips were separated using sharp needles micromanipulated under a stereomicroscope. Then, wires were cut with a sharp blade resulting in an exposed disk metal surface at the tip. At this stage the dual-channel microelectrode sensitivity to $\mathrm{H}_{2} \mathrm{O}_{2}$ was tested. The microelectrodes showing $<20 \%$ difference in $\mathrm{H}_{2} \mathrm{O}_{2}$ sensitivity across channels were selected for coating.

\subsection{Microbiosensor preparation}

Chitosan stock solution at $0.5 \%(\mathrm{w} / \mathrm{v})$ was solubilized in saline $(0.9 \% \mathrm{NaCl})$ under constant stirring during a few hours at $\mathrm{pH} 4-5$, adjusted by addition of $\mathrm{HCl}$. After chitosan solubilisation, the final $\mathrm{pH}$ was set to 5-5.6 by addition of $\mathrm{NaOH}$.

The electrodes were first coated with Nafion by two successive dips in 5\% Nafion solution followed by drying in an oven during $10 \mathrm{~min}$ at $140{ }^{\circ} \mathrm{C}$. This process was repeated four times.

The solution for choline oxidase ( $\mathrm{ChOx}$ ) immobilization was prepared by first adding $1.5 \mathrm{mg}$ of $p$ benzoquinone to $100 \mu \mathrm{L}$ of $0.2 \%$ chitosan. Then a $4 \mu \mathrm{L}$ aliquot of $\mathrm{ChOx}$ at $10 \mathrm{mg} / \mathrm{mL}$ in saline was mixed 
with $4 \mu \mathrm{L}$ of chitosan $/ p$-benzoquinone solution. One of the tips was coated by dipping multiple times (10-15) in a small drop of $\mathrm{ChOx}$ immobilization solution, created using a syringe (Hamilton Co.). The microelectrode and syringe were positioned/moved using micromanipulators under a stereomicroscope and the deposition of coating at the electrode tip was controlled until the chitosan/protein layer was clearly visible under the microscope. The coating procedure was optimized based on preliminary calibrations of biosensors with varying coating thicknesses. Sentinel sites were prepared by adding $0.75 \mathrm{mg} / \mathrm{mL}$ BSA to the coating solution instead of ChOx. The coatings were allowed to cure for at least one hour at room temperature before calibration. After microbiosensor calibration, the distance between wire tips was readjusted to $100-150 \mu \mathrm{m}$ using small needles held by micromanipulators under the stereomicroscope. A small drop of epoxy (Härter GmbH, Germany) was applied at ca. $2 \mathrm{~mm}$ from the wire tips to hold the wires at the desired position.

\subsection{In vitro testing}

The microbiosensors were calibrated by amperometry using an electrochemical system MHS-BR4-VA connected to a 4-channel miniature head-stage (npi electronic GmbH, Germany). The two recording sites were polarized at $+0.7 \mathrm{~V}$ vs. $\mathrm{Ag} / \mathrm{AgCl}$ reference electrode (RE-6, BASi Inc., USA). The DC analog signal from the head-stage was amplified and digitized and stored for offline processing using a multichannel acquisition system (Digital Lynx, Neuralynx Inc., USA).

After stabilization of background current in PBS $(20 \mathrm{~mL})$ for at least $10 \mathrm{~min}$, microelectrodes were calibrated by three consecutive additions of $10 \mu \mathrm{M}$ Ch followed by $4.4 \mu \mathrm{M} \mathrm{H}_{2} \mathrm{O}_{2}$ and $250 \mu \mathrm{M}$ AA. In some calibrations, response to $100 \mu \mathrm{M}$ UA and $1 \mu \mathrm{M}$ DA was also tested. The PBS solution temperature was maintained at $37{ }^{\circ} \mathrm{C}$ using a circulating water pump (Gaymar heating/cooling pump, Braintree Scientific, Inc., USA) connected to the calibration beaker. Calibrations were performed 1-2 days before and after in vivo measurements. Two biosensors were calibrated 1-2 additional times during the weeks following in vivo experiments to evaluate stability over time.

Experiments for evaluation of the oxygen dependence of the microbiosensors response to Ch were carried out at room temperature in a closed beaker. After addition of $5 \mu \mathrm{M} \mathrm{Ch}$, the calibration buffer was bubbled with nitrogen during approximately $30 \mathrm{~min}$. Then, known $\mathrm{O}_{2}$ concentrations were added to the medium from an $\mathrm{O}_{2}$-saturated PBS solution previously bubbled with pure $\mathrm{O}_{2}$ during $20 \mathrm{~min}$.

\subsection{In vivo experiments}

All animal procedures were in accordance with the European Community Council Directive for the Care and Use of Laboratory Animals (86/609/ECC) and German Law for Protection of Animals and were approved by the local authorities. In vivo studies were carried out in 8-9 week old Long-Evans rats (276-325 g), essentially as previously described (Barbosa et al., 2008). Briefly, rats were anesthetized with urethane (1 $\mathrm{g} / \mathrm{kg}$, i.p.) and ketamine/xylazine (80/10 mg/kg i.m.) and placed in a stereotaxic frame (David Kopf Instruments). A high speed rotary (Foredom, Blackstone Industries, Inc., USA) was used to drill a hole in the skull over the brain area of interest. $\mathrm{An} \mathrm{Ag} / \mathrm{AgCl}$ reference electrode ( $70 \mu \mathrm{m}$ diameter), prepared from a 
silver wire, was placed over the skull and fixed in a cotton ball bathed with saline. Recordings were carried out in the CA1 sub-region of the hippocampus. Coordinates relative to bregma were AP -3.4 mm, ML -2.5 $\mathrm{mm}$ and depth relative to brain surface was 2.2-2.4 $\mathrm{mm}$. After background current stabilization, transitions from slow-wave oscillations to theta periods were evoked by tail-pinches.

\subsection{Oxygen measurement in vivo}

In order to simultaneously measure choline and oxygen, the ChOx channel was connected to the npi electrochemical system and polarized at $+0.7 \mathrm{~V}$ vs $\mathrm{Ag} / \mathrm{AgCl}$ as usual. An additional potentiostat was used to measure oxygen reduction on the sentinel channel at $-0.2 \mathrm{~V}$ vs $\mathrm{Ag} / \mathrm{AgCl}$ (EmStat2, PalmSens BV) with an additional $\mathrm{Ag} / \mathrm{AgCl}$ wire to complete the electrochemical cell. The oxygen-measuring channel was calibrated in situ by dividing the current recorded over the brain surface perfused with saline by the oxygen concentration under standard conditions $\left(\left[\mathrm{O}_{2}\right] \approx 250 \mu \mathrm{M}\right)$.

Extracellular oxygen concentration in the brain was modulated by streaming pure oxygen through a mask adapted to the animal's nose (David Kopf Instruments).

\subsection{Data Analysis}

Analysis of calibrations and in vivo recordings was performed using Matlab (MathWorks). Amperometric recordings were acquired at a rate of $32 \mathrm{kHz}$. For response time analysis data were low-pass filtered and down-sampled at $200 \mathrm{~Hz}$. The response time $\left(\mathrm{T}_{50}\right)$ of the sensors was defined as the time interval between the onset of current increase in response to analyte $\left(\mathrm{Ch}\right.$ or $\left.\mathrm{H}_{2} \mathrm{O}_{2}\right)$ and $50 \%$ of the maximum current, as depicted in Figure 1B. For the remaining in vitro analysis data were low-pass filtered at $2 \mathrm{~Hz}$ and down-sampled at 10 Hz.

After sentinel site subtraction (ChOx site - sentinel site), sensitivity to Ch was determined by linearregression of the responses to $\mathrm{Ch}$ additions. The limit of detection (LOD) was calculated as the $\mathrm{Ch}$ concentration corresponding to 3 times the baseline standard deviation (SD). The value of $C h$ sensitivity/ $\mathrm{H}_{2} \mathrm{O}_{2}$ sensitivity $x 100\left(\mathrm{H}_{2} \mathrm{O}_{2}\right.$ sensitivity defined as the ChOx site response to $\mathrm{H}_{2} \mathrm{O}_{2}$ divided by $\mathrm{H}_{2} \mathrm{O}_{2}$ concentration) was used to estimate biosensor efficiency. The selectivity coefficient against AA was calculated from the sentinel subtracted recording as $\log K$, where $K=C h$ sensitivity/AA sensitivity (AA sensitivity equals the absolute value of the response to AA divided by AA concentration).

Since the microbiosensors response to $\mathrm{Ch}$ derives from $\mathrm{ChOx}$ activity, the $\mathrm{O}_{2}$-dependence of the microbiosensors was quantified by calculating the enzymatic parameter apparent $\mathrm{Km}$ for $\mathrm{O}_{2}\left(\mathrm{KmO}_{2}\right)$ under a fixed concentration of $\mathrm{Ch}(5 \mu \mathrm{M})$. The current levels of the sentinel-subtracted recording (relative to baseline before $\mathrm{Ch}$ addition) at the end of the nitrogen bubbling period and after successive additions of known oxygen concentrations were used to calculate the $\mathrm{KmO}_{2}$ and Imax (maximal current), based on the Michaelis-Menten equation. A Generalized Reduced Gradient non-linear algorithm implemented in the Solver function in Microsoft Excel was used to find the $\mathrm{O}_{2}$ concentration at the end of nitrogen bubbling and to fit the Michaelis-Menten equation to the data.

In vivo, amperometric signals reflecting local field potential (LFP) oscillations (Zhang et al., 2009) were 
extracted from the high-frequency component of the sentinel channel amperometric recording $(>0.3 \mathrm{~Hz})$. Data were low-pass filtered and down-sampled at $40 \mathrm{~Hz}$. Power spectrograms were computed using homemade Matlab functions based on multi-taper analysis methods (Mitra and Pesaran, 1999). Theta periods were identified in spectrograms when a prominent band appeared between 2.5-6 Hz (Clement et al., 2008) during $>20 \mathrm{~s}$.

For better visualization of Ch dynamics in vivo, which was slower than LFP oscillations, data were low-pass filtered at $1 \mathrm{~Hz}$ and down-sampled at $10 \mathrm{~Hz}$.

Two strategies were tested for removal of LFP-related currents (common mode rejection) from the Ch recording using the sentinel site signal. First, conventionally used subtraction of the sentinel signal was enhanced by weighting sentinel channel prior to subtraction. The high frequency LFP-related content of the recording, which contributes to most of the noise of choline measurement, was isolated by band-pass filtering (0.3-1 Hz) a portion of the recording (e. g. before a tail pinch). Then a correction factor (wight) as a linear regression coefficient of the $\mathrm{Ch}$ channel current by the sentinel channel current. The residual, cleaned Ch signal, was then calculated as ChOx - sentinel recording $x$ correction factor. The correction factor reduced the effect of small differences in the amplitude of LFP-related currents between channels, which may result from slightly different electrode impedances. However, this procedure does not account for possible phase differences in the complex impedance spectra of electrodes and assumes that amplitude differences are independent of frequency. Therefore, a second sentinel channel subtraction approach was employed by frequency-wise correction of phase and amplitude of the currents of sentinel channel in order to match those of the ChOx channel before subtraction (Woestenburg et al., 1983). This correction was done in frequency domain following Fast Fourier Transformation (FFT), taking into account the following equation:

$$
C h(j w)=\operatorname{ChOx}(j w) \quad T(j w) \operatorname{Sentinel}(j w)
$$

The argument $j w$ denotes a complex value at frequency $w, C h O x(j w)$ and Sentinel(jw) are the FFTs of ChOx and sentinel channel recordings, $T(j w)$ refers to the transfer coefficient which describes the transfer of LFPcurrents from Sentinel to the ChOx channel and $C h(j w)$ represents the FFT of the clean Ch signal. The amplitude of $T$ for each FFT frequency bin was estimated from the square root of ratio between the power of ChOx and sentinel channels. The phase of $T$ was estimated from the phase of the ChOx/sentinel crossspectrum (phase-shift). These estimations were computed for each biosensor used in vivo from average spectra obtained from multiple slow-wave periods. This approach assumes that $\mathrm{Ch}$ fluctuations are largely not coherent with LFP-related currents. Therefore, Ch signals are expected to occur at random phases of LFP oscillations resulting in an average negligible effect on the estimation of the phase of $T$. Importantly, the longer the recording the higher the number of windows for phase-shift calculation, which decreases the interference of $\mathrm{Ch}$ dynamics on the average result. Nevertheless, to reduce this potential interference we considered only the time windows with highest phase locking $(>0.98)$ when calculating the average phaseshift. The presence of $\mathrm{Ch}$ dynamics also increases the power of the $\mathrm{ChOx}$ recording at low frequencies (mainly below $0.3 \mathrm{~Hz}$ ). Since it is not possible to cancel Ch contribution to power, the estimation of the 
amplitude of $T$ in this frequency range was done by linear extrapolation considering the trend at higher frequencies (see example in Figure 4D). Cleaned signal $C h$ was obtained by inverse FFT of $C h(j w)$.

Spectra estimates were obtained by multi-taper spectral analysis (Mitra and Pesaran, 1999), using custommade Matlab functions. Choline amplitude distributions at particular frequencies were calculated from the power spectra of multiple time windows within slow-wave and theta periods. Data is plotted as mean \pm standard error (SEM) and presented in the text and tables as mean \pm standard deviation (SD). Differences between two data sets were evaluated by Student's $t$-test. 


\section{Results}

\subsection{Microbiosensor design}

The microelectrode Pt surface was modified by Nafion coating followed by immobilization of ChOx (or BSA) by covalent binding to chitosan, using $p$-benzoquinone as crosslinker. The scheme in Figure 1A illustrates the biosensor design, as described in the methods section. When assessed by scanning electron microscopy (SEM), the tip surface morphology of the coatings appeared as a rough matrix that covered the entire platinum surface (Figure 1B). According to visual inspection of the wire tips during protein immobilization, the material at the tips represents mainly the chitosan/protein coating.

\subsection{In vitro calibrations}

A representative microbiosensor calibration is shown in Figure 2A. The sensors response to Ch was linear in the standard concentration range tested (up to $30 \mu \mathrm{M}, \mathrm{R}^{2}=0.9999 \pm 0.0001, \mathrm{n}=5$ ). Choline additions induced step current increases at the ChOx site yielding a sensitivity of $324 \pm 46 \mathrm{nA} \mathrm{\mu M}^{-1} \mathrm{~cm}^{-2}(\mathrm{n}=5)$, which is one of the best values reported in the literature using microelectrode array Ch biosensors (Parikh et al., 2004; Zhang et al., 2010). Given the high signal-to-noise ratio of Ch responses, a LOD of $16 \pm 3.6 \mathrm{nM}(\mathrm{n}=5)$ was achieved. Additionally, some calibrations were performed in order to determine the upper limit of the linear dynamic range and the Michaelis-Menten kinetic parameters $\mathrm{Km}$ and maximal response (Imax) of the response to $\mathrm{Ch}$. The sensors response to increasing Ch concentrations followed a saturation trend that fitted to the Michaellis-Menten equation (Figure S1). The average $\mathrm{Km}$ and Imax were $0.4 \pm 0.18 \mathrm{mM}(\mathrm{n}=3)$ and $1.6 \pm 0.5 \mathrm{nA}(\mathrm{n}=3)$. The upper limit of the linear dynamic range was $0.15 \mathrm{mM}(\mathrm{n}=3)$.

Enzyme immobilization was found to be very fast. Responses to Ch were not affected by different curing times tested, from 1 to 24 hours (data not shown). This performance is remarkable considering the difficulties associated with the deposition of enzyme solution on the tip of a $50 \mu \mathrm{m}$ wire as compared to the much larger flat surface of microelectrode arrays (Burmeister et al., 2003; Parikh et al., 2004; Zhang et al., 2010). Indeed, in preliminary tests we found that the method classically used for enzyme immobilization on microelectrode arrays, based on crosslinking by glutaraldehyde in a BSA matrix (Burmeister et al., 2003), was far less effective on the wires than the chitosan-based approach. Crosslinking of chitosan with protein by glutaraldehyde was also tested but the coatings were difficult to control and to reproduce, possibly reflecting the combined effect of the strengthness of this crosslinker and the large number of chitosan's amine groups available.

Immobilized ChOx was highly efficient in translating $\mathrm{Ch}$ into $\mathrm{H}_{2} \mathrm{O}_{2}$ fluxes from bulk solution to the electrode surface. Sensitivities towards $\mathrm{Ch}$ and $\mathrm{H}_{2} \mathrm{O}_{2}$ were of the same order of magnitude, yielding a biosensor efficiency of $60 \pm 11 \%(\mathrm{n}=5)$. This result means that for a given Ch concentration, the ChOx output in steady state produced a $\mathrm{H}_{2} \mathrm{O}_{2}$ flux with a magnitude corresponding to $60 \%$ of the flux generated by externally added $\mathrm{H}_{2} \mathrm{O}_{2}$ at the same concentration. This biosensor efficiency matches the best reported values, obtained with oxidase-based biosensors (O’Neill, 2013). Expectedly, factors like $\mathrm{Ch}$ and $\mathrm{H}_{2} \mathrm{O}_{2}$ diffusion through the coating and $\mathrm{H}_{2} \mathrm{O}_{2}$ loss to the bulk solution limit biosensor efficiency and shape the temporal 
profile of responses to Ch. Comparison of biosensor response profiles to Ch with those to $\mathrm{H}_{2} \mathrm{O}_{2}$ (Figure 2B) suggests that $\mathrm{H}_{2} \mathrm{O}_{2}$ diffuses faster through the coatings, resulting in a shorter $\mathrm{t}_{50}$ response time $(1.4 \pm 0.3 \mathrm{vs}$ $0.52 \pm 0.36 \mathrm{~s}$ for $\mathrm{Ch}$ and $\mathrm{H}_{2} \mathrm{O}_{2}$ respectively, $\mathrm{n}=5, \mathrm{p}<0.05$ ). In the absence of the coating diffusional barrier (bare electrode) the response time to $\mathrm{H}_{2} \mathrm{O}_{2}$ further decreased to $0.25 \pm 0.19 \mathrm{~s}$ ( $\mathrm{n}=37, \mathrm{p}<0.05$ vs coated). In addition to the faster response, bare electrodes were more sensitive to $\mathrm{H}_{2} \mathrm{O}_{2}$ than ChOx-coated sites $(57.7 \pm$ $13.7 \mathrm{pA} / \mu \mathrm{M}, \mathrm{n}=20$ vs $11 \pm 4.0, \mathrm{n}=5, \mathrm{p}<0.05$ ). Modification of the Pt surface by coating layers and diffusion of $\mathrm{Ch}$ and $\mathrm{H}_{2} \mathrm{O}_{2}$ through them apparently are major factors determining the biosensor response profile and sensitivity. Although the experimental setup used does not allow precise determination of response times, the $\mathrm{T}_{50}$ rise time for Ch responses suggests that, similarly to previous reports (Burmeister et al., 2003; Zhang et al., 2010) these microbiosensors are able to follow Ch fluctuations in the time-scale of seconds.

As expected, ChOx and sentinel sites responded similarly to the compounds tested except for $\mathrm{Ch}$, which was not detected by the sentinel site. The response of individual sites to AA, a major electroactive interferent present in the brain extracellular space, was limited by Nafion (Santos et al., 2008). In most cases (4 out of 5) sentinel recording subtraction further improved the biosensor selectivity against AA (see example Figure 2A black vs blue trace) resulting in an average AA selectivity coefficient of $1.9 \pm 0.3(n=5)$. Responses to AA were however more variable than those obtained for $\mathrm{H}_{2} \mathrm{O}_{2}$ ( $p=0.003$, t-test for variances) probably due to the dip-coating procedure used. Selectivity of the biosensors against other potential interferents including uric acid (UA) and dopamine (DA) was tested in some calibrations. Having similar chemical structure, the selectivity coefficient for UA was close to that of AA. The selectivity against DA was the lowest, with an average coefficient around zero, meaning that sensitivities to $\mathrm{Ch}$ and DA are of the same order of magnitude.

In order to evaluate biosensor response stability, calibrations were performed before and after in vivo measurements. No significant drop in sensitivity to Ch was observed after experiments $(6.2 \pm 0.9 \mathrm{pA} / \mu \mathrm{M}$ before vs. $5.9 \pm 1.6 \mathrm{pA} / \mu \mathrm{M}$ after in vivo recordings, $\mathrm{n}=3, p=0.6$ ). Furthermore, two biosensors were calibrated 1-2 additional times during the weeks following brain measurements, covering a total period of eleven days after biosensor preparation. No further drop in sensitivity was observed, thereby supporting the mechanical and chemical robustness of the coatings. The data also suggests the possibility to repeatedly use the same biosensor in multiple in vivo experiments after pre-calibration before each experiment. Table 1 summarizes the analytical properties of the microbiosensors.

\subsection{In vivo measurement of choline}

Cholinergic neurons in the medial septum and diagonal band of Broca (MS/DBB) project to all parahippocampal sub-regions (Teles-Grilo Ruivo and Mellor, 2013). Increased cholinergic tone has been associated with periods of LFP theta oscillations (Hasselmo and McGaughy, 2004; Pignatelli et al., 2011), which provide critical temporal support for information encoding in hippocampus and associated brain regions (Battaglia et al., 2011). Recently, measurements with $\mathrm{Ch}$ microelectrode array biosensors have supported the connection between ACh release in the hippocampus and theta oscillations with unprecedented spatio-temporal resolution. In those studies, increases in extracellular Ch that peaked several seconds after 
theta onset were observed in the hippocampal CA1 sub-region (Zhang et al., 2010).

In light of these findings, we tested the suitability of our approach to measure extracellular choline concentration dynamics during theta and slow-wave periods in the stratum oriens of the CA1 sub-region of the hippocampus. Occurrence of theta periods was either spontaneous or evoked by tail pinch. These periods were identified by spectral analysis of the LFP-related content of the amperometric signal. Figure 3A shows a spectrogram of slow-wave activity spontaneously interrupted by a theta period. The former can be identified by the high power at low frequencies $(<4 \mathrm{~Hz})$ and the latter by the prominent band at approximately $4 \mathrm{~Hz}$. The short recording segments at the bottom show typical current profiles recorded during theta (left) and slow-wave (right) periods. In both cases, the high similarity between channel recordings is evident. However, the same does not apply to longer time-scales. During theta periods, the baseline current increased relative to contiguous slow-wave periods only in the ChOx site, consistent with an increase in extracellular choline.

The presence of the $\mathrm{Ch}$ signal in only one recording site causes a drop in similarity between channel current profiles. Therefore, analysis of spectral coherence was performed to estimate the frequency range of spontaneous Ch fluctuations in vivo. Figure 3C shows a drop in coherence between channels mainly below $0.3 \mathrm{~Hz}$ during slow-wave and theta periods, which is attributable to Ch fluctuations in this range. Accordingly, the power of the $\mathrm{ChOx}$ site recording typically showed a large increase relative to the sentinel in the same frequency range, as exemplified in Figure 3D. Importantly, for higher frequencies the spectra of both power ratio and phase-shift between channels were not constant but rather frequency-dependent (Figure $3 \mathrm{D}$ and $3 \mathrm{E}$ ), possibly owing to differences between the complex impedance spectrum of the electrodes. Considerable variability was however found in the power ratio and phase-shift spectra across biosensors, suggesting that electrode impedance is sensitive to small variations in active area and coating morphology.

Thus, considering the observations above, it is obvious that optimal removal of LFP-related currents from the Ch measurement requires frequency-wise correction of amplitude and phase differences between channels prior to sentinel channel subtraction. As described in the Methods section, this correction was applied to the sentinel recording in the frequency domain by estimating the complex transfer coefficient that describes the transfer of LFP-related currents from sentinel to the ChOx channel. The transfer coefficient $T$ was calculated for each biosensor used in vivo based on power ratio and phase-shift spectra (exemplified in Figure 3).

Sentinel recording subtraction greatly decreased the amplitude of the LFP-related content in the $\mathrm{Ch}$ measurement, improving the signal-to-noise ratio (compare the same recording period in Figure 3B vs 4A). The Ch signal during theta typically reached maximum at about 10-50 seconds after theta dynamics onset. The precise timing of choline peak concentration during theta was variable due to the presence of choline fluctuations with time-courses from few seconds to minutes, which qualitatively agrees with the coherence drop between channels below $0.3 \mathrm{~Hz}$ (Figure 3C). These oscillations were sometimes rhythmic, appearing during both theta and slow-wave periods (recording segments in Figure 4A right).

Compared to a standard sentinel channel subtraction procedure (please see methods section), the phase/amplitude corrected subtraction reduced the noise of Ch recordings, particularly during slow-wave periods, as shown in the recording segments in Figure 4A. A quantitative measure of the LFP-related content 
of both types of subtracted recordings is provided in the average power spectra in Figure 4B, for slow-wave periods with the same biosensor as in panel A. It is clear that, excluding the low frequency range containing Ch dynamics, the power of the subtracted recording upon phase/amplitude correction is much lower than that of the standard-subtracted recording. As expectable, the baseline of the in vitro calibration shows significantly lower power than both traces in vivo, indicating the presence of residual LFP-currents in recordings even following phase/amplitude corrected subtraction. During theta periods, although the absence of slow-waves decreases differences between subtraction approaches, the $4 \mathrm{~Hz}$ theta peak shows much larger power at the standard-subtracted recording (Figure 4C).

The results above underlie the importance of accurate sentinel channel subtraction to improve the signal-tonoise ratio of $\mathrm{Ch}$ recordings in vivo, which avoids bias in Ch signal estimation, especially at shorter time scales, and allows easier identification of spontaneous Ch fluctuations in the nanomolar range. Quantitative data on such fluctuations is provided by the amplitude distributions in Figures 4E and F, obtained from multiple periods in two animals. Both during slow-wave and theta periods, the amplitude of $\mathrm{Ch}$ signals shifted to the right with the signal period. Most fluctuations with $6 \mathrm{~s}$ period had amplitudes around $40 \mathrm{nM}$ whereas the longer signals presented (16 s) frequently had amplitudes around $100 \mathrm{nM}$. Additionally, the dispersion of the distribution increased with signal period, with observations ranging from 20 to nearly 500 $\mathrm{nM}$ for the longer signals.

On top of these fluctuations, larger $\mathrm{Ch}$ changes were observed across states. The amplitude of $\mathrm{Ch}$ increases, relative to slow-wave baseline, following either spontaneous or tail pinch-evoked theta periods ranged between 0.5 and $1 \mu \mathrm{M}$ (Figure 4D, p>0.05 $t$-test between groups).

\subsection{Oxygen dependence of biosensor response}

A potential issue related to the application of oxidase-based biosensors in vivo is their $\mathrm{O}_{2}$ dependence, especially when fluctuations in analyte concentration are in the nanomolar range.

In this work, the $\mathrm{O}_{2}$ dependence of the biosensors was quantified in vitro under a Ch concentration of $5 \mu \mathrm{M}$, which is representative of basal Ch levels in the brain (Garguilo and Michael, 1996; Parikh et al., 2004). The microbiosensor response was modulated by changing the oxygen concentration in the calibration buffer. Nitrogen bubbling after addition of Ch progressively decreased the current recorded at the ChOx site (Figure 5A), indicating a decrease in Ch turnover by ChOx. After current stabilization during nitrogen bubbling, successive additions of oxygen solution induced step current increases, saturating at a value close to the sensor response at normoxic conditions (Figure 5A). The biosensor response as a function of $\mathrm{O}_{2}$ concentration fitted well to a Michaellis-Menten kinetics, as shown in Figure 5B. The $\mathrm{KmO}_{2}$ calculated from the fits averaged $2.6 \pm 1.1 \mu \mathrm{M}(\mathrm{n}=4)$. This result shows that the sensor response is virtually insensitive to changes in $\mathrm{O}_{2}$ above $10 \mu \mathrm{M}$, a value at the low end of the distribution of $\mathrm{O}_{2}$ concentrations found in vivo (Murr et al., 1994; Nair et al., 1987).

\subsection{Effect of oxygen changes on biosensor response in vivo}

In two animals, simultaneous oxygen and choline measurements were performed to confirm the 
independence of the biosensor response towards physiological changes in oxygen concentration predicted by the in vitro tests. Oxygen was measured by polarizing the sentinel site at negative potential whereas the ChOx-coated site potential was at the usual value for Ch measurement.

Exposing the animal to an oxygen-rich atmosphere induced robust increases in extracellular oxygen concentration, which stabilized 43 to 107 seconds ( $n=3$ ) after the beginning of exposure (Figure 6A). As can be observed, a current change in the ChOx site also occurred during this period. However, the increase was much faster than that of oxygen, peaking $12.5 \pm 1.3 \mathrm{~s}(\mathrm{n}=3)$ after exposure started, when oxygen was still near baseline level (Figure 6A). Additionally, the signal in ChOx site showed a pronounced decay during oxygen exposure, reaching values below $40 \%$ of maximal amplitude by the end of exposure period in all trials $(n=3)$. Although the origin of the response at the $\mathrm{ChOx}$ site was not investigated, it possibly represents an authentic cholinergic response to the air-flow at the animal's nose. In agreement with this hypothesis, cholinergic responses have been observed in response to air-puff stimuli in behaving animals (Lovett-Barron et al., 2014).

The uncoupling between choline and oxygen concentration dynamics was also clear following tail-pinch evoked theta. Choline increases evoked by tail-pinch were accompanied either by $\mathrm{O}_{2}$ increases $(\mathrm{n}=1$ out of 5 , Figure $6 \mathrm{~B}$ ) or decreases ( $\mathrm{n}=4$ out of 5, Figure 6C). Furthermore, in both situations faster changes in the ChOx site signal ( $<20 \mathrm{~s}$ period) were not temporally correlated with oxygen dynamics (Figure $6 \mathrm{~B}$ and $\mathrm{C}$ ). These mismatches suggest that, in agreement with the low $\mathrm{KmO}_{2}$ determined in vitro, the biosensor's response in vivo was independent of physiological oxygen changes. 


\section{Discussion}

Recent evidence suggests that the simultaneous tracking of cholinergic activity, neuronal network dynamics and behaviour is crucial to understand the mechanisms of cholinergic neuromodulation (Leão et al., 2012; Lovett-Barron et al., 2014; Parikh et al., 2007; Zhang et al., 2010).

In the present work we have designed a new enzyme-based electrochemical choline microbiosensor to jointly measure cholinergic tone and neuronal network activity using a dual-site disk electrode configuration. The biosensors were successfully applied in the hippocampus of anesthetized rats allowing sensitive measurements of extracellular Ch fluctuations during periods of theta and slow-wave activity. The use of $\mathrm{Pt} / \mathrm{Ir}$ wires as electrode support material is also inexpensive, scalable and compatible with conventional tetrode drives enabling future recordings from multiple brain regions and adjustable dorso-ventral positions in behaving animals.

Previous $\mathrm{Ch}$ measurements in vivo indicated that the concentration dynamics of extracellular $\mathrm{Ch}$ is a reliable index of ACh release under stable acetylcholinesterase activity (Parikh and Sarter, 2013; Parikh et al., 2004). Alternatively, the development of electrochemical biosensors that can directly measure ACh have been reported (Bruno et al., 2006; Burmeister et al., 2008; Mattinson et al., 2011; Mitchell, 2004). This approach relied on differential recordings from acetylcholinesterase/choline oxidase- vs choline oxidase- coated electrode sites. However, reliable distinction between actual $\mathrm{ACh}$ and $\mathrm{Ch}$ is difficult to achieve since small differences in $\mathrm{Ch}$ sensitivities across sites can lead to erroneous attribution of the recorded signal to ACh changes. Indeed, it is still controversial whether these sensors can measure authentic extracellular ACh in vivo (Giuliano et al., 2008). Given these technical issues, the single enzyme design based on ChOx immobilization was adopted in this work.

A distinctive feature of the developed microbiosensors is the very small size of the recording sites $(50 \mu \mathrm{m}$ diameter) and horizontal orientation of the sensor-sentinel pair. This design improves spatial resolution, specially taking into account the cellular organization of cortical and dorsal hippocampal regions where anatomically distinct layers are arranged across the dorso-ventral axis (Anderson et al., 2007). However, faradaic currents on such small electroactive areas are inevitably low which potentially decreases the signalto-noise ratio of measurements. The enzyme immobilization, which should provide high enough enzyme activity to compensate for the small currents, was therefore a key factor to consider on the biosensor design. Choline oxidase was immobilized by covalent binding to chitosan, using $p$-benzoquinone as crosslinker. Chitosan has been widely used in the biosensing field due to its numerous attractive properties such as biocompatibility, high affinity for proteins, excellent film forming ability, good adhesion and versatile functionalization (Honarkar and Barikani, 2009; Krajewska, 2004). Primary amine groups from chitosan and protein molecules can easily undergo nucleophilic reactions with unsubstituted quinones (e. g. $p$ benzoquinone) eventually forming covalent links between amine groups (Nithianandam and Erhan, 1991; Park et al., 2000; Wang et al., 2011; Yang et al., 2009). As a result, it is expectable that the reactions of $p$ benzoquinone with amine groups from chitosan and protein molecules yield a crosslinked network containing combinations of chitosan-chitosan, protein-chitosan and protein-protein bonds. Despite the 
literature knowledge on these reactions, their application in the biosensing field has been scarce. Yet, enzyme immobilization by this method was very fast, robust and provided sensitive responses to $\mathrm{Ch}$. Moreover, the spectra of coherence and amplitude-ratio between channels in vivo indicates that the larger spontaneous $\mathrm{Ch}$ fluctuations (lower coherence) had a much longer period than the biosensors $t_{50}$ for Ch (1.4 s), thereby supporting the reliability of the biosensors to measure spontaneous Ch signals in the time scale of seconds.

The response of individual sites to AA, the major interferent in amperometric measurements in the brain at anodic potentials, was limited by Nafion coating. Selectivity to UA was comparable to that of AA. Due to small size and positive charge selectivity to DA was very low. This is due to poor performance of nafion as compared to phenylenediamine-based coatings (e.g. m-PD) in rejection of DA. Due to small size of our sensor, coating with m-PD was unstable (McMahon et al., 2004), which led to a trade-of between electrode dimension and DA selectivity. However, it is important to take into consideration that DA levels in the hippocampus are much lower than in other brain regions (e. g. striatum), probably in the nanomolar range (Ihalainen et al., 1999; Robinson et al., 2003). Moreover, limited DA selectivity can be generally overcome by our referencing approach using a sentinel site. Whatever the biosensors' selectivity, the absence of responses at the sentinel site concurrent with signals at the ChOx site in vivo (e. g. during theta periods) indicates a negligible interference of these or other electroactive species under the experimental conditions used. Furthermore, the characterization of oxygen dependence of the biosensor response in vitro and in vivo supports a negligible effect of physiological oxygen changes on the recorded signals.

Choline fluctuations lasting few seconds had amplitudes of tens of nanomolar. The detection of such small amplitude events in vivo is generally very difficult to achieve, owing to the presence of LFP-related currents in the recordings (Zhang et al., 2009), which dramatically bias the estimation of the short time scale $\mathrm{Ch}$ fluctuations and increase the noise of electrochemical measurements in vivo as compared to in vitro calibrations. This effect is particularly visible during periods of slow-waves due to their high power at low frequencies (Steriade et al., 1993), close to the scale of Ch dynamics (Figures 3A and B). Thus, although the LFP-related content in electrochemical recordings provides the opportunity to simultaneously assess neuronal network dynamics and neuromodulator tone using a single electrode, the optimization of signal-tonoise ratio of the $\mathrm{Ch}$ measurement requires removal of these currents. When using multi-site biosensors, electrochemical interferents and LFP related currents can be removed from the Ch signal by subtraction of the sentinel channel from the ChOx channel recording (Burmeister et al., 2003; Zhang et al., 2010). However, the arrangement of the sites spanning different depths in previous MEA biosensors was not optimized for cancellation of LFP profiles in most cortical and dorsal-hippocampal regions given the laminar LFP pattern across the dorso-ventral axis in these regions (Buzsáki, 2002; Sharma et al., 2010; Sirota and Buzsáki, 2005). The side-by-side orientation of the recording sites reported here constitutes a more adequate biosensor design for LFP cancellation in these brain regions.

Importantly, in addition to electrode localization, for each frequency the amplitude and phase of LFP-related currents depend on the electrode impedance (Viggiano et al., 2012). Consequently, differences between recording site impedances, that can result from small variations in their active surface area and coating morphology can drop the effectiveness of signal cleaning by sentinel channel subtraction. In order to correct 
for impedance variations across sites, we employed a frequency-wise correction of the phase and amplitude of the sentinel recording to match those of the ChOx channel before subtraction. A complex coefficient $T$ describing the transfer of phases and amplitudes of LFP-related currents from the sentinel into the ChOx channel recordings was estimated from spectra of phase-shift between channels and amplitude ratio, obtained from in vivo recordings. Given the proximity of the recording sites, we hypothesize that $T$ mainly reflects differences between recording sites impedances. Contribution from differences in LFP profiles across sites colocalized to the same anatomical lamina at such low frequencies can be ruled out. The potential interference of $\mathrm{Ch}$ signals in the estimation of $T$ in the low frequency range $(<0.3 \mathrm{~Hz})$ was minimized by selecting only the periods with the highest phase locking between channels and by estimating the power ratio at the range of $\mathrm{Ch}$ dynamics by linear extrapolation from LFP-dominated frequencies. The reliability of this method is supported by comparison of phase/amplitude corrected subtracted vs standard subtracted recordings. Besides decreasing noise, the phase/amplitude correction method does not distort slower $\mathrm{Ch}$ dynamics (Figure 4A), which is corroborated by the similar spectral power of subtracted recordings at the frequency range of Ch fluctuations (Figure 4B and C).

As expected, the power of subtracted recordings at the LFP-dominated range was much lower for phase/amplitude corrected subtractions as compared to standard weighted subtractions (Figure 4B and C), which accounted to the better signal-to-noise ratio of recordings (Figure 4A). Clearly, conventionally used non-weighted substraction (e.g. Zhang et al., 2010) would contaminate Ch dynamics at time scales with significant LFP power. It is feasible, that differences in impedance are mainly evident for small surface area electrodes and become less of a factor with increase of the surface area.

These results demonstrate that the side-by-side biosensor design of minimally invasive recording sites together with the phase/amplitude corrected subtraction procedure can optimize the signal-to-noise ratio and fine localization of in vivo recordings, improving the measurement of local nanomolar Ch dynamics.

Spontaneous Ch fluctuations at time-scales from seconds to minutes were found in vivo. Interestingly, the micromolar $\mathrm{Ch}$ increases during periods of theta oscillations reported here were much higher than those measured in previous choline MEA biosensor recordings in the CA1 sub-region of the hippocampus (Zhang et al., 2010). This mismatch can possibly be related with the lower invasiveness of the biosensors developed in this work which likely damage substantially less cholinergic fibers than the bigger MEAs. Moreover, the higher spatial resolution provided by the small recording sites allows more localized measurements of $\mathrm{Ch}$ changes and thus avoids signal loss due to spatial averaging. In agreement with Zhang and collaborators findings, the increase in ACh release during theta periods described here adds up to previous literature supporting an important role of ACh on the modulation of theta oscillations (Buzsáki, 2002; Pignatelli et al., 2011). However, beyond $\mathrm{Ch}$ changes across states, we also found small amplitude spontaneous $\mathrm{Ch}$ fluctuations during both theta and slow-wave periods. The relationship between these dynamics and LFP (e. g. theta and slow-wave power) in the time-scale of seconds is not straightforward, but the rhythmic patterns sometimes evident in the recordings (Figure 4A) can possibly originate from the interaction between multiple neuron types in the MS/DBB (cholinergic, gabaergic and glutamatergic), suggesting a bi-directional control of ACh release in the hippocampus. These hypotheses are currently under investigation in our lab. 


\section{Conclusions}

The present work reports the development of a biosensor optimized for the joint measurement of neuronal network dynamics and spontaneous Ch fluctuations in the brain in vivo with an effective limit of detection in the nanomolar range. The high spatial-resolution conferred by the small disk-shaped recording sites is specially suited for measurements within small brain regions such as hippocampal sub-layers. The side-byside arrangement of the sites together with an improved method of noise removal by sentinel site subtraction provides high signal-to-noise ratios which allowed the measurement of spontaneous $\mathrm{Ch}$ signals with amplitudes ranging from nanomolar within states to near micromolar in transitions from slow-wave to theta periods.

Future biosensor measurements from multiple brain regions in behaving animals and optogenetic investigation of the neuronal circuits underlying cholinergic signals will help understanding the wide range of Ch dynamics reported here.

\section{Acknowledgements}

This work was supported by the Deutsche Forschungsgemeinschaft (DFG) via Werner Reichardt Centre for Integrative Neuroscience (CIN, Tuebingen), Munich Cluster for Systems Neurology (SyNergy, Munich), Priority Program 1665 and 1392, SFB 654, German Ministry for Education and Research (BMBF) via Grant Number 01GQ0440 (Bernstein Center for Computational Neuroscience Munich), Portuguese Foundation for Science and Technology (FCT) grant PTDC/BBB-BQB/3217/2012. RS acknowledges postdoctoral fellowship SFRH/BPD/82220/2011 from Portuguese Foundation for Science and Technology. 


\section{References}

Anderson, P., Morris, R., Amaral, D., Bliss, T., O’Keefe, J., 2007. The Hippocampus Book, Oxford University Press.

Barbosa, R.M., Lourenço, C.F., Santos, R.M., Pomerleau, F., Huettl, P., Gerhardt, G.A., Laranjinha, J., 2008. In vivo real-time measurement of nitric oxide in anesthetized rat brain. Methods Enzymol. 441, 351367.

Battaglia, F.P., Benchenane, K., Sirota, A., Pennartz, C.M.A., Wiener, S.I., 2011. The hippocampus: Hub of brain network communication for memory. Trends Cogn. Sci.

Bruno, J.P., Gash, C., Martin, B., Zmarowski, A., Pomerleau, F., Burmeister, J., Huettl, P., Gerhardt, G. a, 2006. Second-by-second measurement of acetylcholine release in prefrontal cortex. Eur. J. Neurosci. $24,2749-57$.

Burmeister, J.J., Palmer, M., Gerhardt, G.A., 2003. Ceramic-based multisite microelectrode array for rapid choline measures in brain tissue. Anal. Chim. Acta 481, 65-74.

Burmeister, J.J., Pomerleau, F., Huettl, P., Gash, C.R., Werner, C.E., Bruno, J.P., Gerhardt, G. a, 2008. Ceramic-based multisite microelectrode arrays for simultaneous measures of choline and acetylcholine in CNS. Biosens. Bioelectron. 23, 1382-9.

Buzsáki, G., 2002. Theta Oscillations in the Hippocampus. Neuron 33, 325-340.

Garguilo, M.G., Michael, A.C., 1996. Amperometric microsensors for monitoring choline in the extracellular fluid of brain. J Neurosci Methods 70, 73-82.

Giuliano, C., Parikh, V., Ward, J.R., Chiamulera, C., Sarter, M., 2008. Increases in cholinergic neurotransmission measured by using choline-sensitive microelectrodes: enhanced detection by hydrolysis of acetylcholine on recording sites? Neurochem. Int. 52, 1343-50.

Hasselmo, M.E., McGaughy, J., 2004. High acetylcholine levels set circuit dynamics for attention and encoding and low acetylcholine levels set dynamics for consolidation., in: Progress in Brain Research. pp. 207-31.

Honarkar, H., Barikani, M., 2009. Applications of biopolymers I: Chitosan. Monatshefte fur Chemie 140, 1403-1420.

Ihalainen, J.A., Riekkinen, P., Feenstra, M.G.P., 1999. Comparison of dopamine and noradrenaline release in mouse prefrontal cortex, striatum and hippocampus using microdialysis. Neurosci. Lett. 277, 71-74.

Krajewska, B., 2004. Application of chitin- and chitosan-based materials for enzyme immobilizations: A review. Enzyme Microb. Technol.

Lama, R.D., Charlson, K., Anantharam, A., Hashemi, P., 2012. Ultrafast detection and quantification of brain signaling molecules with carbon fiber microelectrodes. Anal Chem 84, 8096-8101.

Leão, R.N., Mikulovic, S., Leão, K.E., Munguba, H., Gezelius, H., Enjin, A., Patra, K., Eriksson, A., Loew, L.M., Tort, A.B.L., Kullander, K., 2012. OLM interneurons differentially modulate CA3 and entorhinal inputs to hippocampal CA1 neurons. Nat. Neurosci. 15, 1524-30.

Lourenço, C.F., Santos, R.M., Barbosa, R.M., Cadenas, E., Radi, R., Laranjinha, J., 2014. Neurovascular coupling in hippocampus is mediated via diffusion by neuronal-derived nitric oxide. Free Radic. Biol. Med. 73C, 421-429. 
Lovett-Barron, M., Kaifosh, P., Kheirbek, M.A., Danielson, N., Zaremba, J.D., Reardon, T.R., Turi, G.F., Hen, R., Zemelman, B. V, Losonczy, A., 2014. Dendritic inhibition in the hippocampus supports fear learning. Science (80-. ). 343, 857-863.

McMahon, C.P., Killoran, S.J., Kirwan, S.M., O’Neill, R.D., 2004. The selectivity of electrosynthesised polymer membranes depends on the electrode dimensions: implications for biosensor applications. Chem. Commun. (Camb). 2128-2130.

Masamoto, K., Tanishita, K., 2009. Oxygen Transport in Brain Tissue. J. Biomech. Eng. 131, 74002.

Mattinson, C.E., Burmeister, J.J., Quintero, J.E., Pomerleau, F., Huettl, P., Gerhardt, G.A., 2011. Tonic and phasic release of glutamate and acetylcholine neurotransmission in sub-regions of the rat prefrontal cortex using enzyme-based microelectrode arrays. J. Neurosci. Methods 202, 199-208.

McNaughton, B.L., O’Keefe, J., Barnes, C.A., 1983. The stereotrode: A new technique for simultaneous isolation of several single units in the central nervous system from multiple unit records. J. Neurosci. Methods 8, 391-397.

Mitchell, K.M., 2004. Acetylcholine and choline amperometric enzyme sensors characterized in vitro and in vivo. Anal. Chem. 76, 1098-106.

Mitra, P.P., Pesaran, B., 1999. Analysis of dynamic brain imaging data. Biophys J 76, 691-708.

Muñoz, W., Rudy, B., 2014. Spatiotemporal specificity in cholinergic control of neocortical function. Curr. Opin. Neurobiol. 26, 149-60.

Murr, R., Berger, S., Schürer, L., Peter, K., Baethmann, A., 1994. A novel, remote-controlled suspension device for brain tissue PO2 measurements with multiwire surface electrodes. Pflügers Arch. 426, 348 350 .

Nair, P.K., Buerk, D.G., Halsey, J.H., 1987. Comparisons of oxygen metabolism and tissue PO2 in cortex and hippocampus of gerbil brain. Stroke 18, 616-622.

Nithianandam, V.S., Erhan, S., 1991. Quinone-amine polymers: 10. Use of calcium hypochlorite in the syntheses of polyamine-quinone (PAQ) polymers. Polymer (Guildf). 32, 1146-1149.

O’Neill, R., 2013. Characterization of Polymer-Enzyme Composite Biosensors for Brain Monitoring In Vivo, in: Marinesco, S., Dale, N. (Eds.), Microelectrode Biosensors SE - 6, Neuromethods. Humana Press, pp. 115-149.

Parikh, V., Kozak, R., Martinez, V., Sarter, M., 2007. Prefrontal acetylcholine release controls cue detection on multiple timescales. Neuron 56, 141-154.

Parikh, V., Pomerleau, F., Huettl, P., Gerhardt, G.A., Sarter, M., Bruno, J.P., 2004. Rapid assessment of in vivo cholinergic transmission by amperometric detection of changes in extracellular choline levels. Eur. J. Neurosci. 20, 1545-54.

Parikh, V., Sarter, M., 2013. Forebrain Cholinergic Systems and Cognition: New Insights Based on Rapid Detection of Choline Spikes Using Enzyme-Based Biosensors, in: Marinesco, S., Dale, N. (Eds.), Microelectrode Biosensors. Humana Press, pp. 257-277.

Park, S.H., Kwon, O.S., Choi, C.W., Kim, C., 2000. Glucosamine-mediated detoxification of p benzoquinone and its removal by chitosan 21-24.

Pignatelli, M., Beyeler, A., Leinekugel, X., 2011. Neural circuits underlying the generation of theta oscillations. J. Physiol. Paris 106, 81-92. 
Robinson, D.L., Venton, B.J., Heien, M.L.A. V, Wightman, R.M., 2003. Detecting subsecond dopamine release with fast-scan cyclic voltammetry in vivo, in: Clinical Chemistry. pp. 1763-1773.

Santos, R.M., Lourenço, C.F., Piedade, A.P., Andrews, R., Pomerleau, F., Huettl, P., Gerhardt, G.A., Laranjinha, J., Barbosa, R.M., 2008. A comparative study of carbon fiber-based microelectrodes for the measurement of nitric oxide in brain tissue. Biosens. Bioelectron. 24, 704-709.

Sharma, A. V, Wolansky, T., Dickson, C.T., 2010. A comparison of sleeplike slow oscillations in the hippocampus under ketamine and urethane anesthesia. J. Neurophysiol. 104, 932-9.

Sirota, A., Buzsáki, G., 2005. Interaction between neocortical and hippocampal networks via slow oscillations. Thalamus Relat. Syst.

Steriade, M., McCormick, D., Sejnowski, T., 1993. Thalamocortical oscillations in the sleeping and aroused brain. Science (80-. ). 262, 679-685.

Teles-Grilo Ruivo, L.M., Mellor, J.R., 2013. Cholinergic modulation of hippocampal network function. Front. Synaptic Neurosci. 5, 2.

Vandecasteele, M., Varga, V., Berenyi, A., Papp, E., Bartho, P., Venance, L., Freund, T.F., Buzsaki, G., 2014. Optogenetic activation of septal cholinergic neurons suppresses sharp wave ripples and enhances theta oscillations in the hippocampus. Proc. Natl. Acad. Sci. 111, 13535-13540.

Viggiano, A., Marinesco, S., Pain, F., Meiller, A., Gurden, H., 2012. Reconstruction of field excitatory postsynaptic potentials in the dentate gyrus from amperometric biosensor signals. J. Neurosci. Methods 206, 1-6.

Wang, A., Zhang, F., Chen, F., Wang, M., Li, H., Zeng, Z., Xie, T., Chen, Z., 2011. A facile technique to prepare cross-linked enzyme aggregates using p-benzoquinone as cross-linking agent. Korean J. Chem. Eng. 28, 1090-1095.

Woestenburg, J.C., Verbaten, M.N., Slangen, J.L., 1983. The removal of the eye-movement artifact from the EEG by regression analysis in the frequency domain. Biol. Psychol. 16, 127-147.

Yang, X., Liu, Y., Payne, G.F., 2009. Crosslinking Lessons From Biology: Enlisting Enzymes for Macromolecular Assembly. J. Adhes. 85, 576-589.

Zhang, H., Lin, S.-C., Nicolelis, M.A.L., 2010. Spatiotemporal coupling between hippocampal acetylcholine release and theta oscillations in vivo. J. Neurosci. 30, 13431-40.

Zhang, H., Lin, S.C., Nicolelis, M.A., 2009. Acquiring local field potential information from amperometric neurochemical recordings. J Neurosci Methods 179, 191-200. 


\section{Figure legends}

Figure 1. Microbiosensor design. (A) Schematic representation of the biosensor design. Both recording sites were coated with Nafion, which is an exclusion layer against negatively charged molecules (e. g. ascorbate). On top of this layer, the choline-measuring site was coated with a matrix of chitosan (Chit) and choline oxidase (ChOx) crosslinked with $p$-benzoquinone (BQ) whereas the sentinel site (site 2) was coated with a Chit matrix containing bovine serum albumin (BSA). In the presence of $\mathrm{O}_{2}$, ChOx catalyzes choline oxidation to betaine and $\mathrm{H}_{2} \mathrm{O}_{2}$. The latter is oxidized at the platinum surface of site 1. (B) Typical SEM image showing the surface morphology of the coatings.

Figure 2. Microbiosensor calibration. (A) Representative calibration showing responses of $\mathrm{ChOx}$ and sentinel sites to three consecutive additions of $10 \mu \mathrm{M}$ Ch followed by $4.4 \mu \mathrm{M} \mathrm{H}_{2} \mathrm{O}_{2}$ and $250 \mu \mathrm{M} \mathrm{AA}$. The sentinel-subtracted recording is represented by the blue trace. (B) Average normalized temporal profile of responses of bare ( $n=37)$ and Chit/ChOx-coated sites ( $n=5)$ to $4.4 \mu \mathrm{M} \mathrm{H}_{2} \mathrm{O}_{2}$ and of Chit/ChOx-coated sites to the first addition of $10 \mu \mathrm{M} \mathrm{Ch}(\mathrm{n}=5)$. Shadows represent SEM values. The dashed line indicates the response time to $\mathrm{Ch}$ from the average curve, calculated as the time from $\mathrm{Ch}$ addition to $50 \%$ of maximal response (t50).

Figure 3. In vivo recordings of choline and LFP-related content. (A) Power spectrogram from the ChOx channel displaying two periods of slow-wave activity (high power below $4 \mathrm{~Hz}$ ) spontaneously alternating with a period of theta oscillations (prominent power band at $4 \mathrm{~Hz}$ ). Theta and slow-wave current profiles recorded from both channels (low-pass filtered and resampled at $20 \mathrm{~Hz}$ ) at the time-points indicated by the dashed lines are shown at the bottom. (B) Recordings of the period in (A) low-pass filtered at $1 \mathrm{~Hz}$ and resampled at $10 \mathrm{~Hz}$. Sentinel baseline current level was adjusted to match $\mathrm{ChOx}$ site current during slowwave periods, allowing better comparison of current variations during the theta period. (C) Average coherence between ChOx and sentinel channel vs frequency during theta and slow-wave periods ( $\mathrm{n}=15$ for both). (D) The black trace shows the average spectrum of the ratio between Ch1 and Ch2 power obtained from recordings during slow-wave periods ( $\mathrm{n}=8$ periods). The blue trace depicts the corresponding power ratio used to estimate the complex transfer coefficient $T$ for phase/amplitude corrected subtraction. (E) Average spectrum of phase-shift between channels obtained from the same data as (D). Panels A, B, D and E were obtained from recordings using the same biosensor. Data in panel $\mathrm{C}$ was obtained from experiments using two biosensors in different animals.

Figure 4. Sentinel channel subtraction and choline dynamics in vivo. (A) Subtracted recording from data in Figure 3B obtained by phase/amplitude corrected subtraction of sentinel channel to the ChOx channel. The plots on the right correspond to the periods delimited by the dashed squares. Power spectra of subtracted recordings using the same biosensor as in (A) are shown in (B) for slow-wave periods $(n=8)$ and $(C)$ for theta 
periods $(\mathrm{n}=11)$. The recording for the in vitro calibration spectrum in (B) was obtained by standard nonweighted sentinel subtraction. (D) and (E) show normalized amplitude distributions of Ch signals with different periods during slow-wave $(n=15)$ and theta $(n=15)$ periods. (F) Peak amplitudes of choline increases during theta periods relative to contiguous slow-wave periods. Theta periods were either spontaneous $(n=7)$ or evoked by tail pinch $(n=4)$. Data in D-F were obtained from recordings with two biosensors in different animals.

Figure 5. Oxygen dependence of microbiosensor response to choline. (A) Typical sentinel-subtracted responses to changes in $\mathrm{O}_{2}$ concentration in vitro in the presence of Ch. Following addition of $5 \mu \mathrm{M} \mathrm{Ch}$, the calibration buffer was bubbled with nitrogen gas (horizontal bar). At the end of this period a residual $\mathrm{O}_{2}$ concentration remained in solution $\left(\left[\mathrm{O}_{2}\right]_{0}\right)$. Then, known concentrations of $\mathrm{O}_{2}$ were added at the times indicated by the arrows. The rapid transients after $\mathrm{O}_{2}$ additions are artifacts caused by the addition of solution. The current values relative to baseline before $\mathrm{Ch}$ addition corresponding to $\left[\mathrm{O}_{2}\right]_{0}$ and to each $\mathrm{O}_{2}$ addition (after addition artifact) were used to estimate $\left[\mathrm{O}_{2}\right]_{0}$ and the Michaellis-Menten kinetics parameters $\mathrm{KmO}_{2}$ and Imax. The calibration plot of average steady-state current vs. oxygen concentration based on the recording in (A) is shown in (B). Data was fitted to the Michaelis-Menten equation by using non-linear regression analysis.

Figure 6. Measurements of choline and oxygen in vivo. Signals representing choline changes were measured at the ChOx site and $\mathrm{O}_{2}$ was measured simultaneously at the sentinel site polarized at negative potential. (A) Representative $\mathrm{Ch}$ and $\mathrm{O}_{2}$ responses ( $\mathrm{n}=3$ total) recorded following exposure of the animal to a pure $\mathrm{O}_{2}$ stream (horizontal bar). Ch increases following tail-pinch evoked theta (spectrogram not shown, $\mathrm{n}=5$ total) were accompanied by two distinct types of $\mathrm{O}_{2}$ dynamics, illustrated in panels (B) and (C). The transient current changes at the ChOx site during tail-pinch are a stimulus artifacts.

Figure S1. Determination of biosensors Km and Imax for choline. (A) Biosensors were calibrated by successive additions of $\mathrm{Ch}$ until saturation of the response. Arrows indicate the times of $\mathrm{Ch}$ additions at the following concentrations $(\mu \mathrm{M}): 25,25,50,50,125,125,250,250,500,500,500,1000,1000$. (B) The biosensor response was fitted to the Michaellis-Menten equation $\left(\mathrm{R}^{2}=0.989\right)$ for determination of $\mathrm{Km}$ and Imax for Ch. For the fit shown, the Km was $0.3 \mathrm{mM}$ and Imax was $2.1 \mathrm{nA}$. 
Figure 1

FIGURES

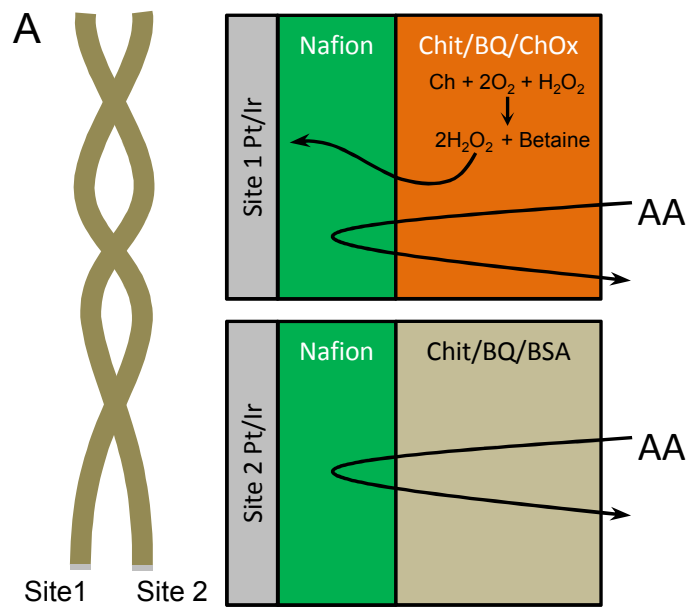

B

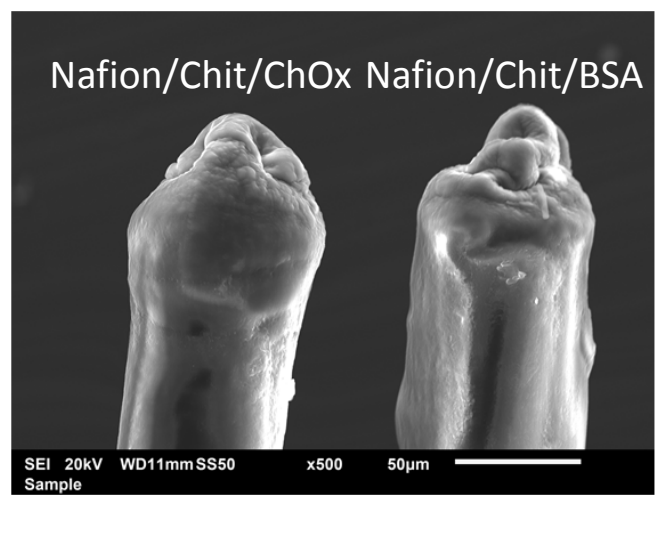


Figure 3

A
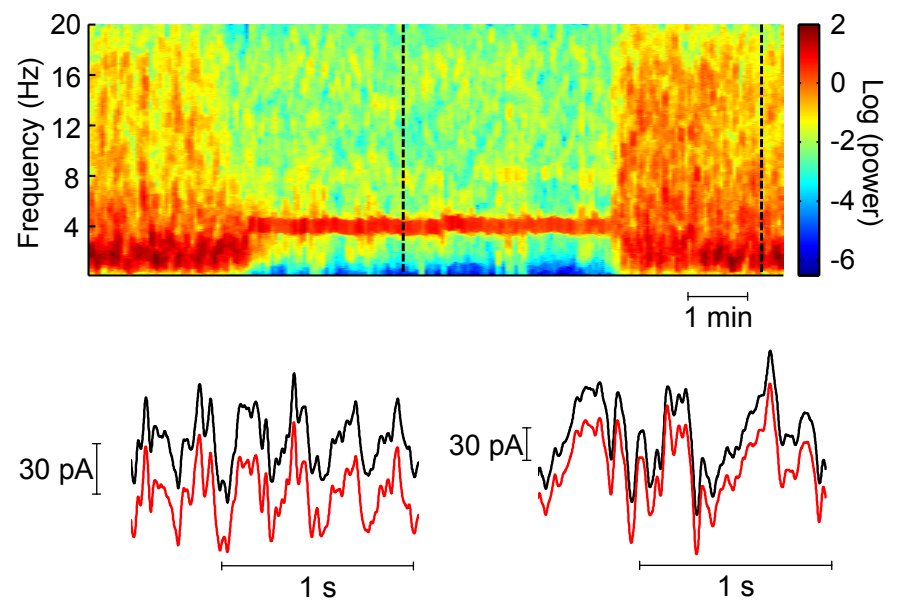

B

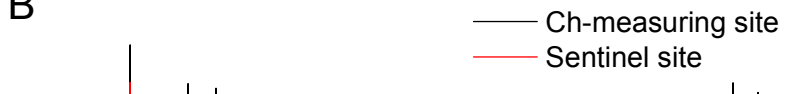

C

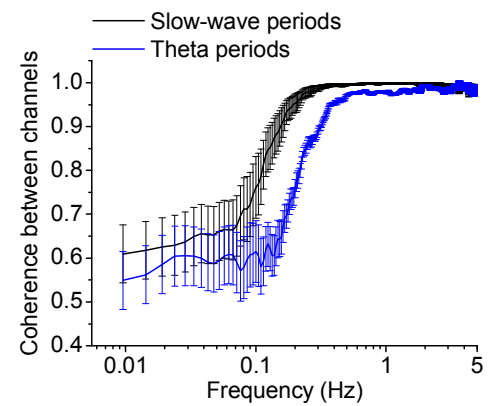

D

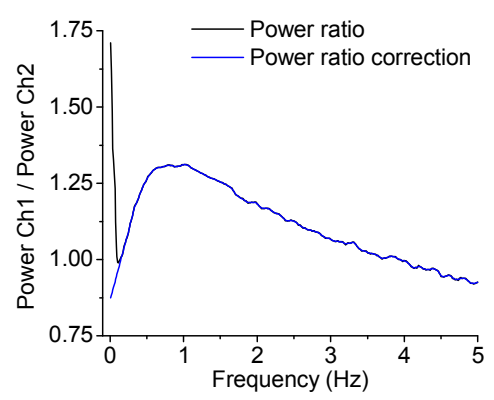

E

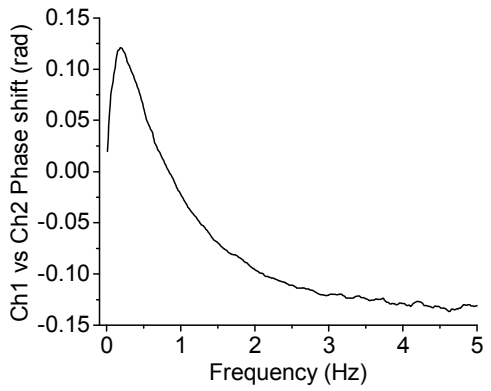



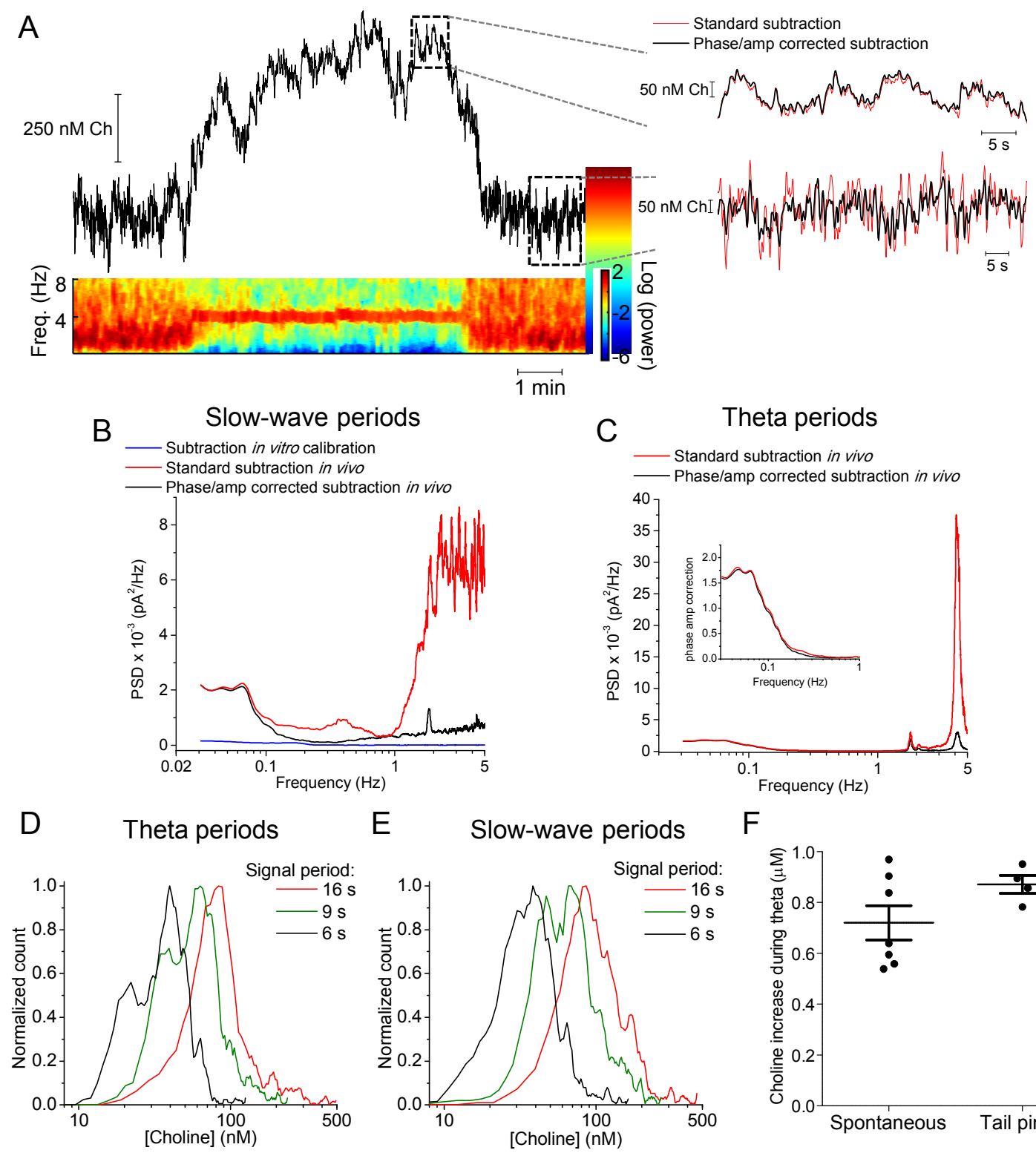

E Slow-wave periods

F
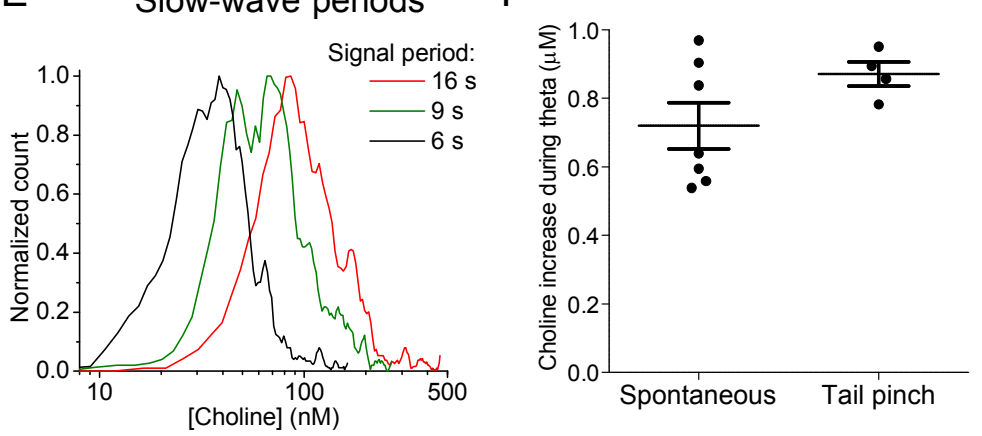


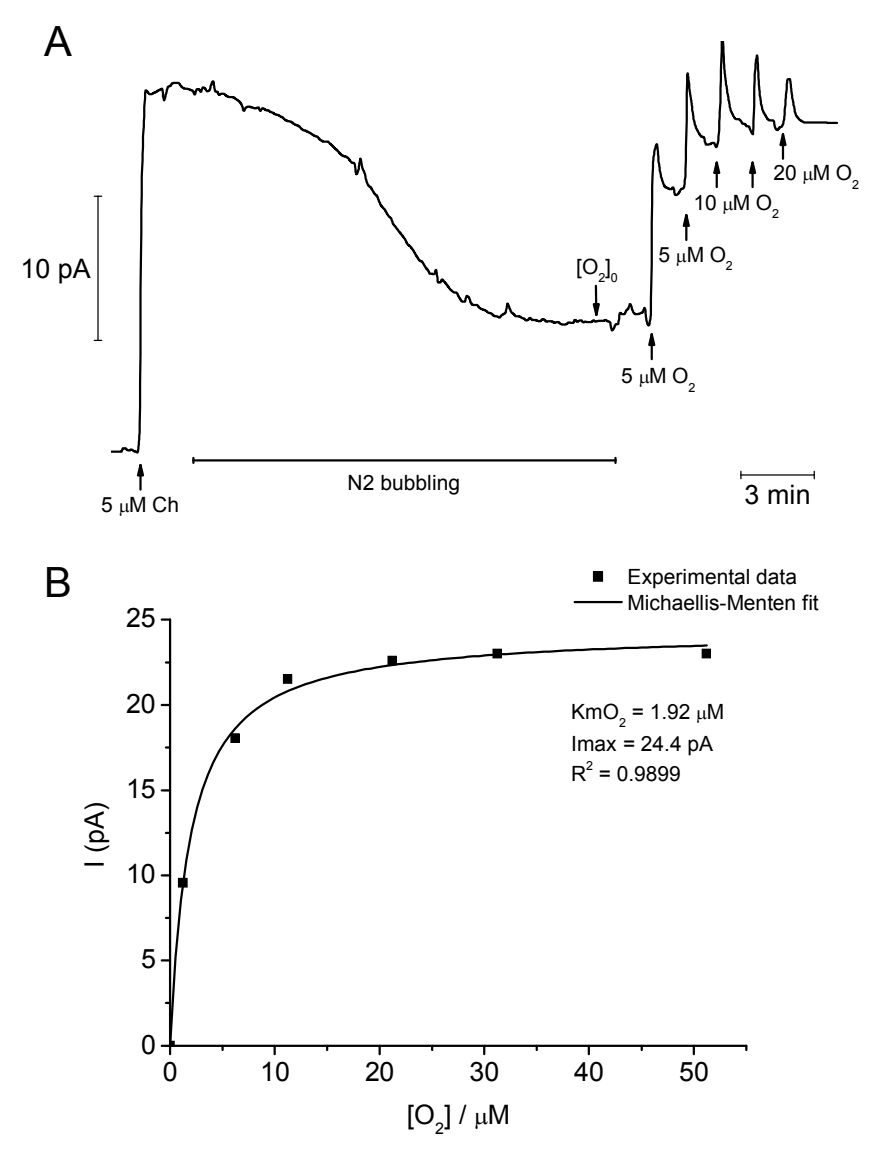

Figure 5

Figure 5
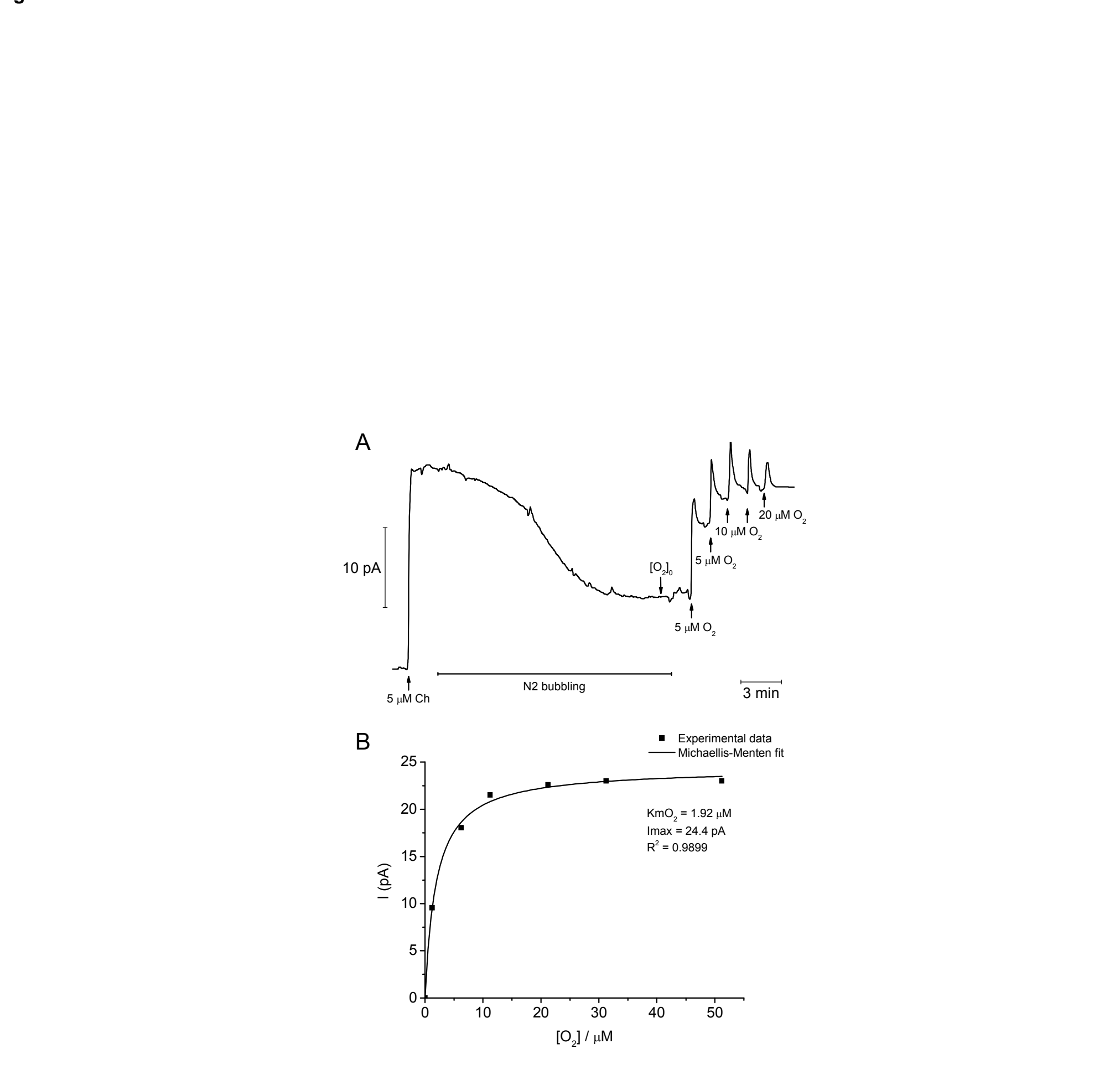

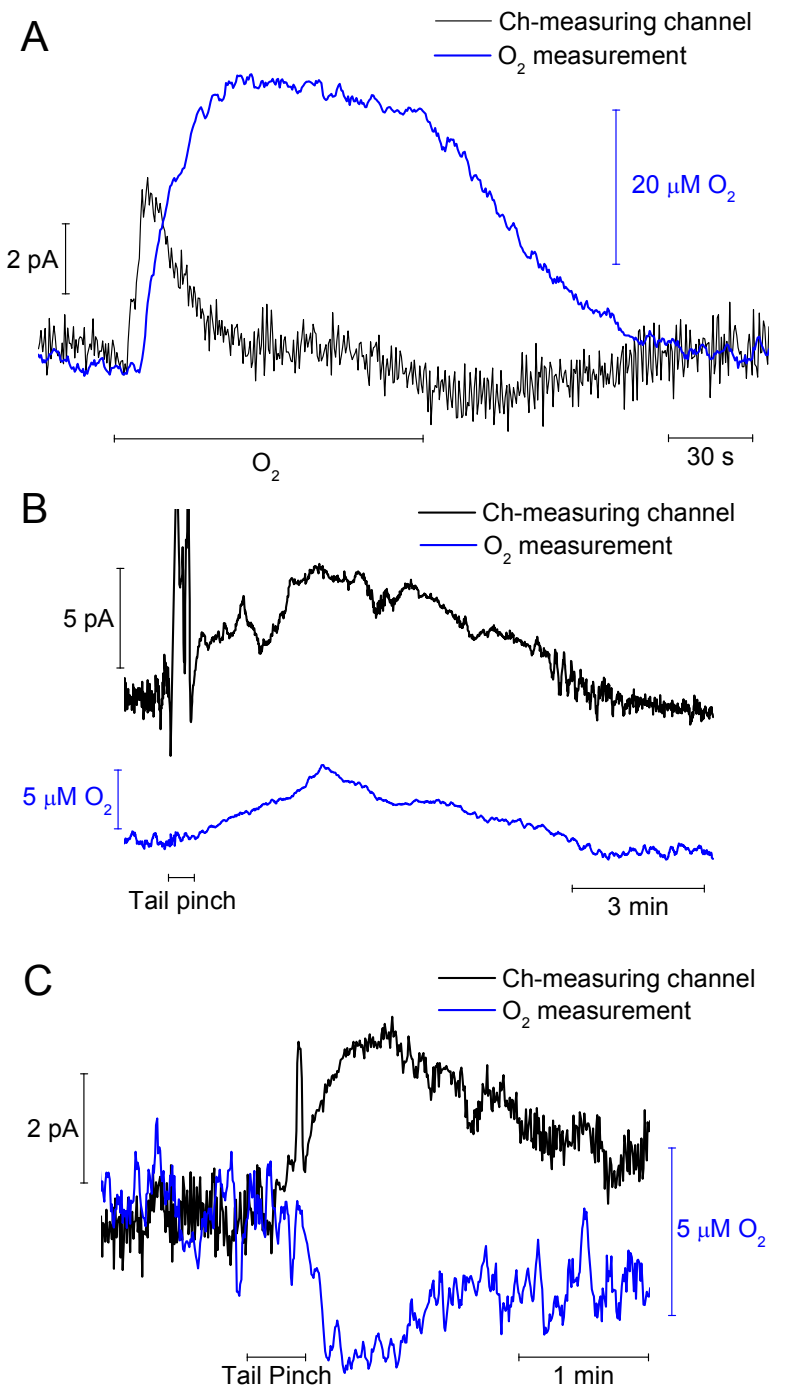
Table 1. Analytical properties of choline microbiosensors

\begin{tabular}{|c|c|c|c|c|c|c|c|c|}
\hline $\begin{array}{c}\text { Sensitivity Ch } \\
(\mathrm{pA} / \mu \mathrm{M})\end{array}$ & $\begin{array}{c}\text { Sensitivity Ch } \\
\left(\mathrm{nA} \mu \mathrm{M}^{-1} \mathrm{~cm}^{-2}\right)\end{array}$ & LOD $(\mathrm{nM})$ & $\begin{array}{c}\text { Sensitivity } \mathrm{H}_{2} \mathrm{O}_{2} \\
(\mathrm{pA} / \mu \mathrm{M})\end{array}$ & $\begin{array}{c}\text { Response } \\
\text { time }(\mathrm{s})\end{array}$ & $\begin{array}{c}\mathrm{KmO}_{2} \\
(\mu \mathrm{M})\end{array}$ & \multicolumn{3}{|c|}{ Selectivity coefficient (log K) } \\
\cline { 5 - 8 } & & & & AA & UA & DA \\
$\begin{array}{c}6.4 \pm 1.2(0.2) \\
(\mathrm{n}=5)\end{array}$ & $\begin{array}{c}324 \pm 46(0.14) \\
(\mathrm{n}=5)\end{array}$ & $\begin{array}{c}16 \pm 3.6(0.23) \\
(\mathrm{n}=5)\end{array}$ & $\begin{array}{c}11 \pm 4.0(0.36) \\
(\mathrm{n}=5)\end{array}$ & $\begin{array}{c}1.4 \pm 0.3 \\
(0.2) \\
(\mathrm{n}=5)\end{array}$ & $\begin{array}{c}2.6 \pm 1.1 \\
(0.41) \\
(\mathrm{n}=4)\end{array}$ & $\begin{array}{c}1.9 \pm 0.3 \\
(0.18) \\
(\mathrm{n}=5)\end{array}$ & $\begin{array}{c}1.75 \pm 0.7 \\
(0.39) \\
(\mathrm{n}=3)\end{array}$ & $\begin{array}{c}-0.18 \pm 0.25 \\
(1.4) \\
(\mathrm{n}=3)\end{array}$ \\
\hline
\end{tabular}

The data are given as the mean \pm SD (RSD).

The number of microbiosensors tested is given in parentesis. 